\title{
Freudenthal duality of near-extremal black holes and Jackiw-Teitelboim gravity
}

\author{
Arghya Chattopadhyay $\odot^{*}$ and Taniya Mandal ${ }^{\dagger}$ \\ National Institute of Theoretical and Computational Sciences, \\ School of Physics and Mandelstam Institute for Theoretical Physics, \\ University of the Witwatersrand, Wits 2050, South Africa
}

(Received 6 December 2021; accepted 3 February 2022; published 23 February 2022)

\begin{abstract}
Freudenthal duality (F-duality), an anti-involution of charge vectors, keeps the entropy and attractor solutions invariant for an extremal supersymmetric black hole. We analyze the effect of F-duality on the entropy of a near-extremal STU black hole in $\mathcal{N}=2$ ungauged, four-dimensional supergravity. We consider double-extremal black holes, whose attractor solutions are fixed in terms of the black hole charges throughout the moduli space. It is well known that Jackiw-Teitelboim (JT) gravity governs the dynamics of the near-horizon regions of higher-dimensional, near-extremal black holes. Owing to this fact, we reduce the four-dimensional supergravity theory to two dimensions to construct a JT gravity-like model and compute the near-extremal entropy. We then analyze the effect of F-duality on this entropy. We show that the F-duality breaks down for the case of near-extremal solutions if one considers the duality operation generated through near-extremal entropy rather than the extremal one.
\end{abstract}

DOI: 10.1103/PhysRevD.105.046014

\section{INTRODUCTION}

Jackiw-Teitelboim (JT) gravity [1,2], a two-dimensional dilaton gravity model has recently become the pursuit of interest for many theoretical physicists, as it provides the simplest playground to study gravitational dynamics. JT gravity, which is basically a particular class of twodimensional dilaton gravity theories, does not have any propagating degrees of freedom. Thus, the effective action crucially depends on the symmetries of the boundary theory. At the boundary of a JT gravity in asymptotically two-dimensional anti-de Sitter $\left(\mathrm{AdS}_{2}\right)$ spacetimes, the time reparametrization symmetry gets slightly broken. As a consequence, the solution to it is given by a nearly $\mathrm{AdS}_{2}$, or $N \mathrm{AdS}_{2}$, spacetime that possesses a nearly onedimensional conformal field theory, or $N \mathrm{CFT}_{1}$, symmetry [3]. Conversely, in the case of a nearly extremal black hole, the near-horizon geometry is given by a $N \mathrm{AdS}_{2}$ geometry which makes a manifest connection between JT gravity and a near-extremal black hole $[3,4]$. Like the nearly extremal black holes, Sachdev-Ye-Kitaev (SYK) models $[5,6]$ have an approximate conformal symmetry $\left(N \mathrm{CFT}_{1}\right)$ in the

\footnotetext{
*arghya.chattopadhyay@wits.ac.za

taniya.mandal@wits.ac.za
}

Published by the American Physical Society under the terms of the Creative Commons Attribution 4.0 International license. Further distribution of this work must maintain attribution to the author(s) and the published article's title, journal citation, and DOI. Funded by SCOAP ${ }^{3}$. infrared limit. This link prompts one to investigate the connection between JT gravity, near-extremal black holes and the SYK model at the low energy limit [7-9]. A suitable modification of JT gravity using higher derivative terms also captures the dynamics of a higher derivative modified, higher-dimensional near-extremal black hole [10,11].

More generally, JT gravity theories defined on any manifold with at least one asymptotic boundary depend on the choice of the boundary curves, and one has to do a path integral over the different choices for these curves as well $[3,8,12]$. These choices of boundary curves or wiggles are governed by the same Schwarzian theory as the low energy limit of the SYK models. Refer to [9] for an excellent review and references therein to further explore this connection. This observation was further explored in [13], showing that the JT gravity path integral on arbitrary orientable surfaces can be computed through ensembles of Hermitian matrices in a particular double scaling limit. This correspondence can also be generalized to different classes of matrix ensembles and JT gravity theories, even JT supergravity theories [14]. The relationship among JT supergravity, the matrix model, and minimal strings, including the nonperturbative effects, has also been analyzed $[15,16]$. The JT model has also been well studied in the context of $N \mathrm{AdS}_{2} / N \mathrm{CFT}_{1}$ holography [17-19].

In this paper, we are interested in studying the behavior of near-extremal black holes using JT gravity as a tool. Extremal black holes have the fascinating property of having finite entropy but zero temperature. An interesting 
phenomenon possessed by the extremal black hole is the attractor mechanism [20,21], in which that the moduli fields always take the charge dependent fixed values at the horizon, regardless of their arbitrary asymptotic values. Thus, the entropy of an extremal black hole can always be written in terms of the black hole charges only. There are numerous works on both supersymmetric and nonsupersymmetric attractors [22,23] which have also been generalized for nonextremal black holes [24,25]. One may wonder whether the properties that hold for an extremal black hole in supergravity also hold true for a nearly extremal black hole. In particular, we are interested in studying the effect of Freudenthal duality (F-duality) on near-extremal black holes. Freudenthal triple systems have long been known to mathematicians due to Hans Freudenthal [26], and they were then connected to the entropy of a supersymmetric black hole [27]. In the case of four-dimensional extremal, supersymmetric black holes, the entropy and corresponding attractor solutions are invariant under an anti-involutive operation of charges. This discrete duality is called Freudenthal duality [26-36]. It differs from U-duality (electromagnetic duality), as the quantities which remain invariant under U-duality, except for entropy and attractor solutions, change with Freudenthal duality.

In [37], it was explicitly shown that for $\mathcal{N}=2$, type IIA supergravity, an extremal supersymmetric $D 0-D 4-D 6$ black hole is Freudenthal dual to an extremal supersymmetric $D 0-D 2-D 4-D 6$ black hole. Using the H-FGK [extension of FGK (Ferrara-Gibbons-Kallosh) formalism with an extra variable $\mathrm{H}$ ] formalism [38], it was shown in [39] that Freudenthal duality behaves as part of a local symmetry for the cases of both extremal and nonextremal black holes in $N=2, d=4$ ungauged supergravity. Freudenthal duality has also been studied in the context of $\mathcal{N}>2$ supergravities [31]. In this paper, we consider the STU model in four-dimensional, ungauged, $\mathcal{N}=2$ type IIA supergravity in asymptotically flat space, with all possible dyonic black hole charges. Entropy of the extremal, supersymmetric black hole solution in this model is Freudenthal invariant. Here we explicitly check to determine whether this duality is preserved in the case of a near-extremal black hole in the context of a JT-like theory that we get after dimensionally reducing the higherdimensional theory. Asymptotically flat, rotating, nonextremal black hole solutions were studied in [40-42]. Our final aim is to study F-duality for these generic nonextremal cases; this work takes the initial step in that direction by studying near-extremal versions.

In asymptotically flat space, the near-horizon region of a higher-dimensional extremal (or near-extremal) black hole contains a throatlike region with $\mathrm{AdS}_{2} \times S^{2}$ geometry, whereas the far-horizon region is asymptotically flat. One of the solutions to the equations of motion that come from the effective action [43] after dimensional reduction is
$\mathrm{AdS}_{2}$ with a constant dilaton $\Phi_{0}$ [45]. We call this the background solution. It can be easily checked to see that this background $\mathrm{AdS}_{2}$ solution is the same as that appearing in the near-horizon limit of the higher-dimensional extremal black hole and $\Phi_{0}$ is the radius of both $\mathrm{AdS}_{2}$ and $S^{2}$. To go beyond extremality, we introduce a perturbation around this background solution. Expanding the reduced effective action around this fluctuation, we find a JT-like action. Following [3], one can then find the entropy of the near-extremal black hole in terms of the value of the dilaton fluctuation at the boundary-namely, $\phi_{b}$. As we are interested in the nature of higher-dimensional physics, we fix the boundary at the overlapping region of near-horizon and far-zone geometries in a higher-dimensional black hole solution $[44,46,47]$. At this boundary, the dilaton fluctuation takes a fixed value given by black hole charges. Thus, we can effectively fix the entropy of a near-extremal black hole in terms of the black hole charges, where we consider the charges to remain fixed while moving away from extremality.

Semiclassically, at low temperatures, the mass difference between the extremal state and the near-extremal state $\Delta M=M-M_{\text {ext }}$ scales as $\Delta M \sim \frac{T^{2}}{M_{\text {gap }}}$ for a fixed charge black hole. Here $M$ and $T$ denote the mass and the temperature of the near-extremal state and $M_{\text {ext }}$ is the mass of the extremal state of the black hole. This calls the reliability of the semiclassical analysis into question, as the black hole does not radiate at the temperature $T \lesssim M_{\text {gap }}$, since the energy required to Hawking radiate is of the order of the temperature $T$. Thus, previously it was believed that there was a mass gap between the extremal state and the near-extremal state in the mass spectrum at very low temperature when a fixed charge black hole was studied semiclassically. Including quantum effects, it was shown in [44] that for a nonsupersymmetric black hole mass gap can be removed, thus enabling black holes to Hawking radiate at any nonzero temperature. As an effect, the entropy of a near-extremal black hole achieves an extra term, logarithmic in $\left(T \phi_{b}\right)$, in addition to a term linear in $T \phi_{b}$. Conversely, in [47] it was shown that there is a mass gap for a near-extremal, near-Bogomol'nyi-PrasadSommerfield (near-supersymmetric) black hole solution in $\mathcal{N}=2$ pure four-dimensional, ungauged supergravity by matching with $\mathcal{N}=4$ super-JT gravity. In fact, the existence of a mass gap is justified in this case as the degeneracy of the black hole is consistent with the Bekenstein-Hawking entropy formula [47]. However, in our current context, we are looking at the entropy of a nearextremal black hole in fermionic truncated supergravity; thus, there is no mass gap in our black hole spectrum once we include quantum corrections.

Reserving the details for the main text, in a simplified form, F-duality for black holes can be understood as an anti-involution mapping of the charge vector $\mathcal{Q}^{\mathcal{A}}$ as 


$$
F: \hat{\mathcal{Q}}^{\mathcal{A}} \rightarrow \Omega_{\mathcal{A B}} \frac{\partial S_{0}(\mathcal{Q})}{\partial \mathcal{Q}^{\mathcal{B}}}
$$

with $\Omega_{\mathcal{A B}}$ being some symplectic metric and $S_{0}(\mathcal{Q})$ is the extremal, supersymmetric entropy. Under this mapping $S_{0}(Q)$ remains invariant $\left[S_{0}(\hat{\mathcal{Q}})=S_{0}(\mathcal{Q})\right]$. We show that for a double-extremal attractor solution (for which the values of the moduli fields remain fixed in terms of the charges throughout the moduli space) for a fourdimensional ungauged STU model the near-extremal entropy takes the following form:

$$
S_{\mathrm{NE}}=S_{0}+\mathcal{A} T S_{0}^{\frac{3}{2}}+\frac{3}{2} \log \left(\mathcal{B} T S_{0}^{\frac{3}{2}}\right)
$$

where $T$ is the temperature of the black hole and $\mathcal{A}$ and $\mathcal{B}$ are charge and temperature independent constants. We analyze the effect of Freudenthal duality on this form of near-extremal entropy. We show that $S_{\mathrm{NE}}$ is not invariant under F-duality if one takes the full entropy $S_{\mathrm{NE}}$ as the function generating the F-dualization. The F-duality invariance of black holes instead always has to be defined through the extremal entropy through Eq. (1.1), in which case $S_{\mathrm{NE}}$ remains invariant.

We have structured the paper as follows. In Sec. II, we lay out a bare-bones description of the bosonic sector of the $\mathcal{N}=2$ supergravity and F-duality. We show the nearextremal entropy calculations in Sec. III, following a dimensional reduction of the full four-dimensional metric to derive an effective two-dimensional dilaton gravity theory. In Sec. IV, we discuss the fate of F-duality for the near-extremal scenario, and we finally conclude in Sec. V. In the Appendix, we tabulate the necessary details of the STU black hole and the attractor solutions.

\section{BRIEF REVIEW OF $\mathcal{N}=2$ SUPERGRAVITY AND F-DUALITY}

In four dimensions, the bosonic part of the $\mathcal{N}=2$ supergravity action coupled with an arbitrary number of vector multiplets is given by

$$
\begin{aligned}
S= & \frac{-1}{8 \pi G_{4}} \int d^{4} x \sqrt{-G}\left(-\frac{R}{2}+h_{a \bar{b}} \partial_{\mu} x^{a} \partial_{\nu} x^{\bar{b}} G^{\mu \nu}\right. \\
& \left.-\mu_{\Lambda \Sigma} \mathcal{F}_{\mu \nu}^{\Lambda} \mathcal{F}_{\lambda \rho}^{\Sigma} G^{\mu \lambda} G^{\nu \rho}-\nu_{\Lambda \Sigma} \mathcal{F}_{\mu \nu}^{\Lambda} * \mathcal{F}_{\lambda \rho}^{\Sigma} G^{\mu \lambda} G^{\nu \rho}\right),
\end{aligned}
$$

where $G_{\mu \nu}$ is the spacetime metric with the Ricci scalar $R$ and determinant G. $x^{a}$ are the $n$ complex moduli scalar fields with the moduli space metric $h_{a \bar{b}}$. The field strength $\mathcal{F}^{\Lambda}$ corresponds to the $(n+1)$ one-form $A_{\mu}^{\Lambda}$, $\Lambda=0,1, \ldots, n$. The gauge coupling constants $\mu_{\Lambda \Sigma}$ and $\nu_{\Lambda \Sigma}$ and the moduli space metric are determined by the prepotential $F$. The number of vector multiplets is denoted by $n$ and $G_{4}$ denotes the four-dimensional Newton's constant.

We are interested in a four-dimensional, type IIA $\mathcal{N}=2$ supergravity that arises at the low energy limit when type IIA string theory is compactified on a Calabi-Yau manifold $\mathcal{M}$ in the large volume limit. This theory is described by a holomorphic function called the prepotential that is given by

$$
F=D_{a b c} \frac{X^{a} X^{b} X^{c}}{X^{0}}
$$

where the symplectic section $X^{a}$ is related to the complex moduli as $x^{a}=\frac{X^{a}}{X^{0}}$. $D_{a b c}$ is the triple intersection number $D_{a b c}=\frac{1}{6} \int_{\mathcal{M}} \alpha_{a} \wedge \alpha_{b} \wedge \alpha_{c}$ of two-form $\alpha_{a}$, the basis of the cohomology group $H^{2}(\mathcal{M}, Z)$. The Kahler potential is given by

$$
K=-\log \left[i \sum_{\Lambda=0}^{n}\left(\overline{X^{\Lambda}} \partial_{\Lambda} F-X^{\Lambda} \partial_{\Lambda}^{-} F\right)\right]
$$

which can be further simplified using the gauge $X^{0}=1$ as follows:

$$
K=-\log \left[-i D_{a b c}\left(x^{a}-\bar{x}^{a}\right)\left(x^{b}-\bar{x}^{b}\right)\left(x^{c}-\bar{x}^{c}\right)\right] .
$$

The moduli space metric can be constructed as $h_{a \bar{b}}=\partial_{a} \partial_{\bar{b}} K$, i.e., by taking the partial derivative of the Kahler metric $K$ with respect to the complex moduli $x^{a}$. The gauge coupling constants are given by the real and imaginary parts of $\mathcal{N}$ as $\mu=\operatorname{Im}(\mathcal{N})$ and $\nu=-\operatorname{Re}(\mathcal{N})$, where

$$
\mathcal{N}=\overline{F_{\Lambda \Sigma}}+2 i \frac{\operatorname{Im}\left(F_{\Lambda \Omega}\right) \operatorname{Im}\left(F_{\Pi \Sigma}\right) X^{\Omega} X^{\Pi}}{\operatorname{Im}\left(F_{\Omega \Pi}\right) X^{\Omega} X^{\Pi}}
$$

and $F_{\Lambda \Sigma}=\partial_{\Lambda} \partial_{\Sigma} F$.

In this paper, for simplicity, we consider the STU model where the number of coupled vector multiplets is $n=3$ and the only nonzero intersection matrix is $D_{123}=1 / 6$. For a $D 0-D 2-D 4-D 6$ black hole in STU supergravity, the entropy of an extremal, supersymmetric black hole is given by [48]

$$
S_{\text {STU,susy }}^{D 0-D 2-D 4-D 6}=\frac{\pi}{p^{0}} \sqrt{\frac{2}{3 d_{123}} \Delta_{1} \Delta_{2} \Delta_{3}-\left(p^{0}(p . q)-2 D\right)^{2}},
$$

where $\Delta_{a}=D_{a b c} \tilde{x}^{b} \tilde{x}^{c}=3 D_{a b c} p^{b} p^{c}-p^{0} q_{a}$ with a real $\tilde{x}^{a}$ and $D=D_{a b c} p^{a} p^{b} p^{c}$. Following [37], one can write the above expression as 
$S_{\text {STU,susy }}^{D 0-D 2-D 4-D 6}=\frac{\pi}{3 p^{0}} \sqrt{\frac{4\left(3 D-p^{0} q_{a} p^{a}\right)^{3}}{D}-9\left(p^{0}(p \cdot q)-2 D\right)^{2}}$,

where $p . q=p^{0} q_{0}+p^{a} q_{a} \cdot q_{0}, p^{0}, q_{a}$ and $p^{a}$ are the $D 0$-, $D 6-, D 2-$, and $D 4$-brane charges. As mentioned in [49], for a generic supergravity model, there could be more than one set of supersymmetric attractor solution with different forms of entropies for different charge sectors, depending on the value of the involutory matrix $I_{b}^{a}$. The properties of $I_{b}^{a}$ are

$$
I_{c}^{a} I_{b}^{c}=\delta_{b}^{a} \quad \text { and } \quad D_{a d e} I_{b}^{d} I_{c}^{e}=D_{a b c} .
$$

For the STU model, there is only one choice of the matrix where $I_{b}^{a}=\delta_{b}^{a}$. Thus, in the STU model we have only one expression for the entropy for all possible $D$-brane charges.

Now we will briefly summarize F-duality, which is a nonlinear, anti-involutive transformation of charge vector $\mathcal{Q}^{M}=\left(p^{\Lambda}, q_{\Lambda}\right)$ in which the entropy and the attractor values of an extremal, supersymmetric black hole remain fixed under this transformation. The transformation acts as follows:

$$
\begin{gathered}
\pi \hat{\mathcal{Q}}^{M}=\Omega^{M N} \frac{\partial S(\mathcal{Q})}{\partial \mathcal{Q}^{N}}, \\
\hat{\hat{\mathcal{Q}}}=-\mathcal{Q}, \quad \text { and } \quad S(\hat{\mathcal{Q}})=S(\mathcal{Q}),
\end{gathered}
$$

where $S(\mathcal{Q})$ is the extremal entropy of a supersymmetric black hole corresponding to the charge vector $\mathcal{Q}^{M}=$ $\left(p^{\Lambda}, q_{\Lambda}\right) \cdot \Omega^{M N}$ is $2(n+1) \times 2(n+1)$ symplectic matrix with $\Omega^{T}=-\Omega$ and $\Omega^{2}=-I$. We consider $\Omega=\left(\begin{array}{cc}0 & -I \\ I & 0\end{array}\right)$.

Thus, by F-dualizing an extremal $D 0-D 2-D 4-D 6$ supersymmetric black hole, one would expect to get another extremal $D 0-D 2-D 4-D 6$ supersymmetric black hole whose charges nonlinearly depend on that of the former one.
Nevertheless, an extremal $D 0-D 2-D 4-D 6$ supersymmetric black hole can also be mapped to an extremal $D 0-D 4-D 6$ supersymmetric black hole via F-duality [37].

\section{JT-LIKE THEORY FROM $D=4, \mathcal{N}=2$ SUPERGRAVITY}

Extremal black holes are interesting objects in both classical gravity and supergravity by their own virtues. In both cases, the near-horizon geometry of a fourdimensional extremal, charged black hole in asymptotically flat space factorizes as $\mathrm{AdS}_{2} \times S^{2}$ with the same radius for $\mathrm{AdS}_{2}$ and $S_{2}$. In this section, we show that, starting with a supergravity theory in four dimensions, we find a JT-like dilaton gravity theory in two dimensions upon dimensional reduction. This JT-like theory captures the dynamics of the near-extremal black hole solution of the supergravity theory, where the extremal solution that we are interested in is supersymmetric. It has been seen that, for an extremal black hole in supergravity, irrespective of whether or not it is supersymmetric, the entropy is fixed in terms of black hole charges. Also, the values of the moduli fields are fixed in terms of charges at the horizon, we call them attractor solutions. We are interested in the fate of F-duality once we perturb away from the extremal scenario.

\section{A. Dimensional reduction}

We start with a dimensional reduction on the sphere at the near-horizon region by considering a spherically symmetric ansatz for the metric such as $[50,51]$

$$
d s^{2}=G_{\mu \nu} d x^{\mu} d x^{\nu}=\tilde{g}_{\alpha \beta} d x^{\alpha} d x^{\beta}+\Phi^{2} d \Omega_{2}^{2} .
$$

We are interested in dyonic black hole solutions, i.e., having both electric charges $Q_{\Lambda}$ and magnetic charges $P^{\Lambda}$. Dimensionally reducing the bulk action over Eq. (3.1) while further assuming that $\mathcal{F}_{\theta \phi}^{\Lambda}=P^{\Lambda} \sin \theta$, we find

$$
\begin{aligned}
\tilde{S}_{\text {bulk }}= & -8 \pi G_{4} S_{\text {bulk }}=4 \pi \int d^{2} x \sqrt{-\tilde{g}} \Phi^{2}\left[-\frac{R(\tilde{g})}{2}-\frac{1}{\Phi^{2}}\left(\nabla_{\alpha} \Phi\right)^{2}-\frac{1}{\Phi^{2}}-\mu_{\Lambda \Sigma} \mathcal{F}_{\alpha \beta}^{\Lambda} \mathcal{F}^{\Sigma \alpha \beta}-\frac{2}{\Phi^{4}} \mu_{\Lambda \Sigma} P^{\Lambda} P^{\Sigma}\right] \\
& -4 \pi \int d^{2} x\left(4 \nu_{\Lambda \Sigma} P^{\Lambda} \mathcal{F}_{r t}^{\Sigma}\right)+8 \pi \int d^{2} x \sqrt{\tilde{g}} \nabla_{\alpha}\left(\Phi \nabla^{\alpha} \Phi\right)+4 \pi \int d^{2} x \sqrt{-\tilde{g}} \Phi^{2} h_{a \bar{b}} \partial_{\alpha} x^{a} \partial_{\beta} x^{\bar{b}} \tilde{g}^{\alpha \beta}
\end{aligned}
$$

One should note that the scalar fields $x^{a}$ only have a dependence on the radial direction. For our convenience, we write this reduced action in the following way by introducing a two-dimensional Levi-Civita symbol $\epsilon^{\alpha \beta}$ such that $\epsilon^{r t}=1$ :

$$
\begin{aligned}
\tilde{S}_{\text {bulk }}= & 4 \pi \int d^{2} x \sqrt{-\tilde{g}} \Phi^{2}\left[-\frac{R(\tilde{g})}{2}-\frac{1}{\Phi^{2}}\left(\nabla_{\alpha} \Phi\right)^{2}-\frac{1}{\Phi^{2}}-\mu_{\Lambda \Sigma} \mathcal{F}_{\alpha \beta}^{\Lambda} \mathcal{F}^{\Sigma \alpha \beta}-\frac{2}{\Phi^{4}} \mu_{\Lambda \Sigma} P^{\Lambda} P^{\Sigma}\right] \\
& -4 \pi \int d^{2} x\left(2 \nu_{\Lambda \Sigma} P^{\Lambda} \epsilon^{\alpha \beta} \mathcal{F}_{\alpha \beta}^{\Sigma}\right)+8 \pi \int d r d t \sqrt{\tilde{g}} \nabla_{\alpha}\left(\Phi \nabla^{\alpha} \Phi\right)+4 \pi \int d^{2} x \sqrt{-\tilde{g}} \Phi^{2} h_{a \bar{b}} \partial_{\alpha} x^{a} \partial_{\beta} x^{\bar{b}} \tilde{g}^{\alpha \beta}
\end{aligned}
$$


This two-dimensional action contains a derivative on the dilation $\Phi$. As our target is to get a JT-like theory, we do not want a term like that in the two-dimensional action. A simple way to get rid of that is Weyl rescaling of the metric in the following way:

$$
g_{\alpha \beta}=\frac{\Phi}{\Phi_{0}} \tilde{g}_{\alpha \beta}
$$

The Weyl rescaled bulk action is then given by

$$
\begin{aligned}
\tilde{\mathcal{S}}_{\text {bulk }}= & 4 \pi \int d^{2} x \sqrt{-g} \Phi^{2}\left[-\frac{R(g)}{2}-\frac{\Phi_{0}}{\Phi^{3}}-\frac{\Phi}{\Phi_{0}} \mu_{\Lambda \Sigma} \mathcal{F}_{\alpha \beta}^{\Lambda} \mathcal{F}^{\Sigma \alpha \beta}-\frac{2 \Phi_{0}}{\Phi^{5}} \mu_{\Lambda \Sigma} P^{\Lambda} P^{\Sigma}\right] \\
& -4 \pi \int d^{2} x\left(2 \nu_{\Lambda \Sigma} P^{\Lambda} \mathcal{F}_{\alpha \beta}^{\Sigma} \epsilon^{\alpha \beta}\right)+6 \pi \int d^{2} x \sqrt{-g} \nabla_{\alpha}\left(\Phi \nabla^{\alpha} \Phi\right)+4 \pi \int d^{2} x \sqrt{-g} \Phi^{2} h_{a \bar{b}} \partial_{\alpha} x^{a} \partial_{\beta} x^{\bar{b}} g^{\alpha \beta} .
\end{aligned}
$$

We also need to dimensionally reduce the GibbonsHawking-York (GHY) boundary term and the boundary term for the gauge field as well. From Eq. (2.1), one can check to see that the three-dimensional GHY boundary term corresponding to $-8 \pi G_{4} S$ is given by

$$
S_{\mathrm{GHY}}^{(3)}=-\int d^{3} x \sqrt{H} K^{(3)},
$$

where $H$ is the induced hypersurface metric of the full metric $G$ and $K^{(3)}$ is the three-dimensional extrinsic curvature tensor. Defining $h_{\mu \nu}$ as the induced metric and $\hat{K}$ as the reduced extrinsic curvature from the reduced 1D point of view, one can write

$$
S_{\mathrm{GHY}}=-4 \pi \int \sqrt{-h} \Phi^{2}\left(\hat{K}+\frac{2}{\Phi} \hat{n} \cdot \nabla \Phi\right),
$$

where $\hat{n}_{\alpha}$ is the unit normal vector on the hypersurface. After Weyl rescaling, this can be rewritten as

$$
\mathcal{S}_{\mathrm{GHY}}=-4 \pi \int \sqrt{-h} \Phi^{2}\left(K+\frac{3}{2 \Phi} n \cdot \nabla \Phi\right),
$$

where $n$ is the Weyl rescaled unit vector and $K$ is the corresponding extrinsic curvature. A three-dimensional boundary term needed for the well behaved variation of $-8 \pi G_{4} S$ with respect to the gauge field is given by [52]

$S_{\text {gauge }}^{(3)}=4 \int d^{3} x \sqrt{-H}\left[\mu_{\Lambda \Sigma} n_{\mu} \mathcal{F}^{\Lambda \mu \nu} A_{\nu}^{\Sigma}+\nu_{\Lambda \Sigma} n_{\mu} * \mathcal{F}^{\Lambda \mu \nu} A_{\nu}^{\Sigma}\right]$.

After dimensional reduction and the Weyl transformation, this term can be written as

$$
\begin{aligned}
\mathcal{S}_{\text {gauge }}= & 16 \pi \int d t \sqrt{-h} \frac{\Phi^{3}}{\Phi_{0}} \mu_{\Lambda \Sigma} n_{\alpha} \mathcal{F}^{\Lambda \alpha \beta} A_{\beta}^{\Sigma} \\
& +16 \pi \int d t \frac{\sqrt{-h}}{\sqrt{-g}} \nu_{\Lambda \Sigma} \frac{\Phi^{2}}{\Phi_{0}^{2}} n_{r} P^{\Lambda} A_{t}^{\Sigma} .
\end{aligned}
$$

Thus, the full dimensionally reduced, Weyl rescaled action is

$$
\begin{aligned}
\mathcal{S}_{\text {tot }}= & -4 \pi \int d^{2} x \sqrt{-g}\left[\frac{\Phi^{2} R(g)}{2}+\frac{\Phi_{0}}{\Phi}+\frac{\Phi^{3}}{\Phi_{0}} \mu_{\Lambda \Sigma} \mathcal{F}_{\alpha \beta}^{\Lambda} \mathcal{F}^{\Sigma \alpha \beta}+\frac{2 \Phi_{0}}{\Phi^{3}} \mu_{\Lambda \Sigma} P^{\Lambda} P^{\Sigma}-\Phi^{2} h_{a \bar{b}} \partial_{\alpha} x^{a} \partial_{\beta} x^{\bar{b}} g^{\alpha \beta}\right] \\
& -4 \pi \int d^{2} x\left(2 \nu_{\Lambda \Sigma} P^{\Lambda} \mathcal{F}_{\alpha_{\beta}}^{\Sigma} \epsilon^{\alpha^{\beta}}\right)+16 \pi \int d t \sqrt{-h}\left[\frac{\Phi^{3}}{\Phi_{0}} \mu_{\Lambda \Sigma} n_{\alpha} \mathcal{F}^{\Lambda \alpha \beta} A_{\beta}^{\Sigma}+\frac{1}{\sqrt{-g}} \nu_{\Lambda \Sigma} n_{r} P^{\Lambda} A_{t}^{\Sigma}\right]-4 \pi \int \sqrt{-h} \Phi^{2} k .
\end{aligned}
$$

Corresponding dilaton and gravity equations of motion are

$$
\begin{gathered}
\Phi R(g)-\frac{\Phi_{0}}{\Phi^{2}}+3 \frac{\Phi^{2}}{\Phi_{0}} \mu_{\Lambda \Sigma} \mathcal{F}_{\alpha \beta}^{\Lambda} \mathcal{F}^{\Sigma \alpha \beta}-6 \frac{\Phi_{0}}{\Phi^{4}} \mu_{\Lambda \Sigma} P^{\Lambda} P^{\Sigma}-2 \Phi h_{a \bar{b}} \partial_{\alpha} x^{a} \partial_{\beta} x^{\bar{b}} g^{\alpha \beta}=0 \\
-\frac{1}{2} g_{\gamma \delta}\left[-\frac{\Phi^{2} R}{2}-\frac{\Phi_{0}}{\Phi}-\frac{\Phi^{3}}{\Phi_{0}} \mu_{\Lambda \Sigma} \mathcal{F}_{\alpha \beta}^{\Lambda} \mathcal{F}^{\Sigma \alpha \beta}-\frac{2 \Phi_{0}}{\Phi^{3}} \mu_{\Lambda \Sigma} P^{\Lambda} P^{\Sigma}+\Phi^{2} h_{a \bar{b}} \partial_{\alpha} x^{a} \partial_{\beta} x^{\bar{b}} g^{\alpha \beta}\right]-\frac{\Phi^{2}}{2} R_{\gamma \delta} \\
-\frac{2 \Phi^{3}}{\Phi_{0}} \mu_{\Lambda \Sigma} \mathcal{F}_{\delta \beta}^{\Lambda} \mathcal{F}_{\gamma \psi}^{\Sigma} g^{\beta \psi}+\Phi^{2} h_{a \bar{b}} \partial_{\gamma} x^{a} \partial_{\delta} x^{\bar{b}}-\nabla_{\gamma} \Phi \nabla_{\delta} \Phi-\Phi \nabla_{\gamma} \nabla_{\delta} \Phi+\left(\left(\nabla^{\mu} \Phi\right)^{2}+\Phi \nabla^{2} \Phi\right) g_{\gamma \delta}=0 .
\end{gathered}
$$


We also have equations for gauge fields and the moduli fields. As our case of interest is the double-extremal solution; i.e., when the moduli fields take a constant value in terms of the black hole charges throughout the moduli space, equations corresponding to them are trivially satisfied. Consequently Eq. (3.12) and a trace of Eq. (3.13) simplify to

$$
\begin{gathered}
\Phi R(g)-\frac{\Phi_{0}}{\Phi^{2}}+3 \frac{\Phi^{2}}{\Phi_{0}} \mu_{\Lambda \Sigma} \mathcal{F}_{\alpha \beta}^{\Lambda} \mathcal{F}^{\Sigma \alpha \beta}-6 \frac{\Phi_{0}}{\Phi^{4}} \mu_{\Lambda \Sigma} P^{\Lambda} P^{\Sigma}=0 \\
\frac{\Phi_{0}}{\Phi}-\frac{\Phi^{3}}{\Phi_{0}} \mu_{\Lambda \Sigma} \mathcal{F}_{\alpha \beta}^{\Lambda} \mathcal{F}^{\Sigma \alpha \beta}+\frac{2 \Phi_{0}}{\Phi^{3}} \mu_{\Lambda \Sigma} P^{\Lambda} P^{\Sigma} \\
+\left(\nabla_{\mu} \Phi\right)^{2}+\Phi \nabla^{2} \Phi=0 .
\end{gathered}
$$

Remembering that the gauge field equation of motion coming out of the full 4D theory has the following property in the near-horizon limit,

$$
\partial_{\mu}\left(\sqrt{g} \Phi^{2} \mu_{\Lambda_{\Sigma}} \mathcal{F}^{\Sigma \mu \nu}+\nu_{\Lambda_{\Sigma}} P^{\Sigma} \epsilon^{\mu \nu}\right)=0,
$$

the solution to the above equation can be written as

$$
\sqrt{g} \Phi^{2} \mu_{\Lambda_{\Sigma}} \mathcal{F}^{\Sigma \mu \nu}+\nu_{\Lambda_{\Sigma}} P^{\Sigma} \epsilon^{\mu \nu}=-Q_{\Lambda} \epsilon^{\mu \nu}
$$

with the usual convention $\epsilon^{r t}=-\epsilon^{t r}=1$ and $\epsilon_{r t}=g \epsilon^{r t}$. For convenience, we rescale the electric and magnetic charges as $P^{\Lambda}=\sqrt{\frac{G_{4}}{2}} p^{\Lambda}$ and $Q_{\Lambda}=\sqrt{\frac{G_{4}}{2}} q_{\Lambda}$. Then in two dimensions, following Eq. (3.17), the solution to the gauge field equation takes the following form:

$\sqrt{g} \frac{\Phi^{3}}{\Phi_{0}} \mu_{\Lambda_{\Sigma}} \mathcal{F}^{\Sigma \mu \nu}+\sqrt{\frac{G_{4}}{2}} \nu_{\Lambda_{\Sigma}} p^{\Sigma} \epsilon^{\mu \nu}=-\sqrt{\frac{G_{4}}{2}} q_{\Lambda} \epsilon^{\mu \nu}$.

To get a JT-like action, we integrate out the gauge fields from Eq. (3.11). The effective theory is given by the following action:

$$
\begin{aligned}
\mathfrak{S}= & 4 \pi \int d^{2} x \sqrt{-g}\left[-\frac{\Phi^{2} R}{2}-\frac{\Phi_{0}}{\Phi}\right. \\
& \left.-\frac{2 \Phi_{0}}{\Phi^{3}}\left(\mathcal{X}+\frac{G_{4}}{2} \mu_{\Lambda \Sigma} p^{\Lambda} p^{\Sigma}\right)\right] \\
& -4 \pi \int d t \sqrt{-h} \Phi^{2} k
\end{aligned}
$$

where

$$
\begin{aligned}
\mathcal{X}\left(\mu_{\Lambda, \Sigma}, \nu_{\Lambda, \Sigma}, P, Q\right)= & \frac{G_{4}}{2}\left(\left(\mu^{-1}\right)^{\Lambda \Sigma} q_{\Lambda} q_{\Sigma}\right. \\
& +p^{\Sigma}\left(\nu \mu^{-1}\right)_{\Sigma}{ }^{\Lambda} q_{\Lambda}+q_{\Lambda}\left(\mu^{-1} \nu\right)^{\Lambda} p^{\Sigma} \\
& \left.+p^{\Lambda}\left(\nu \mu^{-1} \nu\right)_{\Lambda \Sigma} p^{\Sigma}\right) .
\end{aligned}
$$

We can also get Eq. (3.19) by using the on-shell value (3.18) of the gauge field strength in Eq. (3.11). The equations of motion derived from this effective action are

$$
\begin{array}{r}
\Phi R(g)-\frac{\Phi_{0}}{\Phi^{2}}-6 \frac{\Phi_{0}}{\Phi^{4}}\left(\mathcal{X}+\frac{G_{4}}{2} \mu_{\Lambda \Sigma} p^{\Lambda} p^{\Sigma}\right)=0 \\
1+\frac{2}{\Phi^{2}}\left(\mathcal{X}+\frac{G_{4}}{2} \mu_{\Lambda \Sigma} p^{\Lambda} p^{\Sigma}\right)+\frac{\Phi}{\Phi_{0}}\left(\nabla_{\mu} \Phi\right)^{2}+\frac{\Phi^{2}}{\Phi_{0}} \nabla^{2} \Phi=0 .
\end{array}
$$

This alternate action can be simply viewed as some 2D dilaton theory as $\mathfrak{S}=\mathfrak{S}_{\text {bulk }}+\mathfrak{S}_{\text {bdy }}$, where

$$
\begin{aligned}
& \mathfrak{\Im}_{\text {bulk }}=-\frac{1}{2 G_{4}} \int d^{2} x \sqrt{-g}\left[-\frac{\Phi^{2} R}{2}-U(\Phi)\right], \\
& \mathfrak{\Im}_{\text {bdy }}=\frac{1}{2 G_{4}} \int d t \sqrt{-h} \Phi^{2} K,
\end{aligned}
$$

with the effective dilaton potential $U(\Phi)=\frac{\Phi_{0}}{\Phi}+$ $\frac{2 \Phi_{0}}{\Phi^{3}}\left(\mathcal{X}+\frac{G_{4}}{2} \mu_{\Lambda \Sigma} p^{\Lambda} p^{\Sigma}\right)$. We have inserted the normalization $\frac{-1}{8 \pi G_{4}}$ in front of the action so as to carefully track the gravitational constant from now on.

One of the solutions to Eq. (3.21) is given by

$$
\begin{aligned}
\Phi & =r, \text { with } d s^{2}=\frac{\Phi}{\Phi_{0}}\left(-f(r) d t^{2}+\frac{d r^{2}}{f(r)}\right) \\
f(r) & =1+\frac{\mathcal{C}}{\Phi}+\frac{2 \Theta(\mathcal{Q})}{\Phi^{2}}, \quad \Theta(\mathcal{Q})=-\left(\mathcal{X}+\frac{G_{4}}{2} \mu_{\Lambda \Sigma} p^{\Lambda} p^{\Sigma}\right),
\end{aligned}
$$

where $\mathcal{C}$ is an integration constant, which we shall fix shortly. As elaborately explained in [46] and further addressed in [44,47], it is always beneficial to consider the near-horizon and the far-horizon regions of the four-dimensional, near-extremal black hole spacetime separately. The near-horizon $(\mathrm{NH})$ region can be well approximated as $\mathrm{AdS}_{2} \times S^{2}$. This approximation holds true in the region where the radial distance $r-r_{0} \ll r_{0}$, with $r_{0}$ being the horizon radius for the extremal case. The farhorizon $(\mathrm{FH})$ metric remains well approximated by the extremal case even in the nonextremal scenario, and it is given by

$$
d s^{2}=-f(r) d t^{2}+\frac{d r^{2}}{f(r)}+r^{2} d \Omega_{2}^{2} .
$$


In the extremal limit, near the horizon, the metric (3.24) reads as

$d s^{2}=-\frac{\left(r-r_{0}\right)^{2}}{L_{2}^{2}} d t^{2}+\frac{L_{2}^{2}}{\left(r-r_{0}\right)^{2}} d r^{2}+r_{0}^{2} d \Omega_{2}^{2}, \quad L_{2}=r_{0}$,

whereas the solution (3.23) takes the following form:

$d s^{2}=-\frac{\left(r-r_{0}\right)^{2}}{L_{2}^{2}} d t^{2}+\frac{L_{2}^{2}}{\left(r-r_{0}\right)^{2}} d r^{2}, \quad \Phi=r_{0}$.

Using this blackening factor as the boundary condition, one can now fix the integration constant in Eq. (3.23) as $\mathcal{C}=-2 r_{0}$ in the large $r$ limit. Also, in this limit, $\Theta(Q)=\frac{r_{0}}{2}$. Both of these regions overlap inside the bulk where the radial distance satisfies the condition that the $\mathrm{AdS}_{2}$ radius $L_{2} \ll r-r_{0} \ll r_{0}$. One should note at this point that in the present context we are dealing with an asymptotically flat four-dimensional metric, and therefore for our purposes $L_{2}=r_{0}=\Phi_{0}$ for the extremal situation. Interestingly, if one considers a holographic screen at an arbitrary radial distance $r=r_{0}+r_{b}$ inside the overlapping $\mathrm{NH}$ and $\mathrm{FH}$ regions, the leading low temperature behavior is independent of the choice of $r_{b}$ [44,46]. In the following, we will use this crucial aspect to fix the near-extremal entropy as a function of the dyonic charges.

\section{B. Near-extremal entropy}

Thus far, we have focused only on the near-horizon region and have dimensionally reduced the action corresponding to Eq. (2.1) on $S^{2}$. We have also derived the twodimensional, effective dilaton gravity action (3.22). At the horizon, dilaton $\Phi$ takes the constant value $r_{0}$, which gets fixed from Eq. (3.21) as

$$
r_{0}^{2}=2 \Theta(\mathcal{Q}), \quad R=-\frac{2}{\Phi_{0}^{2}} .
$$

In the near-horizon region where $r-r_{0} \ll r_{0}$, the values of the dilaton and the metric are given by small perturbation around the background solution, i.e., a constant dilaton and $\mathrm{AdS}_{2}$ metric. In two dimensions, the metric can be written in terms of a single independent parameter. In conformal gauge we write the metric as

$$
d s^{2}=e^{2 \omega}\left(-d t^{2}+d \rho^{2}\right) .
$$

For the background solution we set $\omega=\omega_{0}$. Using the coordinate transformation

$$
\frac{L_{2}^{2}}{\rho}=r-r_{0}
$$

where $r_{0}$ is the horizon of extremal solution, and identifying $L_{2}=r_{0}$ one can write the two-dimensional background metric (3.26) with a constant dilaton as

$$
d s^{2}=e^{2 \omega_{0}}\left(-d t^{2}+d \rho^{2}\right), \quad e^{2 \omega_{0}}=\frac{L_{2}^{2}}{\rho^{2}},
$$

where $\rho \equiv \rho(r)$ is a function of the original radial coordinate. For Eq. (3.28) the Ricci scalar boils down simply to

$$
R=-2 e^{-2 \omega}\left(\frac{\partial^{2} \omega}{\partial \rho^{2}}-\frac{\partial^{2} \omega}{\partial t^{2}}\right)
$$

Therefore, the bulk part of the dilaton action (3.22) can be written for Eq. (3.28) and a generic dilaton $\Phi$ as

$\mathfrak{\Im}_{\text {bulk }}=\frac{1}{2 G_{4}} \int d^{2} x \sqrt{-g}\left[\Phi^{2} e^{-2 \omega}\left(\frac{\partial^{2} \omega}{\partial \rho^{2}}-\frac{\partial^{2} \omega}{\partial t^{2}}\right)-U(\Phi)\right]$.

The effective potential $U(\Phi)$ vanishes for the background constant dilaton solution (3.26). To evaluate the complete action (3.22), one can now switch to the Euclidean time $\tau=i t$ and simply write the extrinsic curvature scalar as $K=1 / r_{0}$. The complete action (3.22) for this background solution provides the extremal entropy. Using the attractor functional method [53], one can see that the extremal entropy is

$$
S_{0}=\frac{\pi r_{0}^{2}}{G_{4}}=\frac{2 \pi|\Theta(\mathcal{Q})|}{G_{4}} .
$$

In terms of charges, it takes the following explicit form [21]:

$$
\begin{aligned}
S_{0}= & \frac{\pi}{2} \mid\left(\mu^{-1}\right)^{\Lambda \Sigma} q_{\Lambda} q_{\Sigma}+p^{\Sigma}\left(\nu \mu^{-1}\right)_{\Sigma}{ }^{\Lambda} q_{\Lambda}+q_{\Lambda}\left(\mu^{-1} \nu\right)^{\Lambda} p^{\Sigma} \\
& +p^{\Lambda}\left(\nu \mu^{-1} \nu\right)_{\Lambda \Sigma} p^{\Sigma}+\left.\mu_{\Lambda \Sigma} p^{\Lambda} p^{\Sigma}\right|_{\text {horizon }} .
\end{aligned}
$$

In the above formula, the values of $\mu_{\Lambda \Sigma}$ and $\nu_{\Lambda \Sigma}$ have to be evaluated at the horizon using the attractor values and Eq. (2.5). For an STU black hole with all possible $D$-brane charges in type IIA supergravity, one can use the attractor values pointed out in the Appendix and rewrite the entropy as [48]

$S_{\mathrm{STU}}^{D 0-D 2-D 4-D 6}=\frac{\pi}{p^{0}} \sqrt{\frac{2}{3} \Delta_{1} \Delta_{2} \Delta_{3}-\left(p^{0}(p \cdot q)-2 D\right)^{2}}$.

It was shown in [37] that $D 0-D 2-D 4-D 6$ black hole has a Freudenthal dual black hole with charge configuration $D 0-D 4-D 6$. One can further show that an STU $D 0-D 2-D 4-D 6$ black hole can also be F-dual to another $D 0-D 2-D 4-D 6$ black hole.

We will now switch on the perturbation around the dilaton and the metric as 


$$
\Phi=r_{0}(1+\phi), \quad \omega=\omega_{0}+\Omega .
$$

With this scheme of perturbation $\mathbb{\mathfrak { S }}$ can be written as $\mathfrak{S}_{\text {bulk }}=\mathfrak{S}_{\text {bulk }}^{0}+\mathfrak{S}_{\text {bulk }}^{1}+\mathfrak{S}_{\text {bulk }}^{2}+\mathcal{O}\left(\phi^{3}\right)$, where

$$
\begin{aligned}
& \mathfrak{\Im}_{\text {bulk }}^{0}=\frac{1}{2 G_{4}} \int d^{2} x \sqrt{-g}\left[r_{0}^{2} e^{-2 \omega}\left(\frac{\partial^{2} \omega}{\partial \rho^{2}}-\frac{\partial^{2} \omega}{\partial t^{2}}\right)-U\left(\Phi_{0}\right)\right], \\
& \mathfrak{\Im}_{\text {bulk }}^{1}=\frac{\Phi_{0}^{2}}{2 G_{4}} \int \sqrt{g}\left(-\phi(t, z)\left(-\frac{R}{2}-\frac{1}{\Phi_{0}^{2}}\right)\right) .
\end{aligned}
$$

The boundary action can also be written as a power series of $\phi$ and $\Omega$ as $\mathfrak{S}_{\text {bdy }}=\mathfrak{S}_{\text {bdy }}^{0}+\mathfrak{S}_{\text {bdy }}^{1}+\mathfrak{S}_{\text {bdy }}^{2}+\mathcal{O}\left(\phi^{3}\right)$. As stated earlier, $\mathfrak{S}_{\text {bulk }}^{0}+\mathfrak{S}_{\text {bdy }}^{0}$ provides the extremal entropy. Using Eq. (3.27), we see that $\mathfrak{S}_{\text {bulk }}^{1}$ vanishes and we are left with the boundary action $\mathfrak{S}_{\text {bdy }}^{1}$ linearly in $\phi$. In a Euclidean signature, the boundary action is

$$
\mathfrak{S}_{\text {bdy }}^{1}=-\frac{r_{0}^{2}}{G_{4}} \int d x \sqrt{h} \phi K
$$

To evaluate the above integral, we consider the curve separating the near-horizon and far-horizon regions of a four-dimensional, near-extremal black hole as our boundary. On this boundary, the induced metric is $h=\frac{L_{2}^{2}}{\epsilon^{2}}$ and the proper length is $\int d u \sqrt{h}=\frac{\beta L_{2}}{\epsilon}$. Here, $\beta$ is the periodicity of the boundary time $u$ and the constant boundary value of the dilaton is $\left.\phi\right|_{\text {bdy }}=\frac{\phi_{b}}{\epsilon}$. This boundary cuts out a patch of near-horizon $\mathrm{AdS}_{2}$ space. In Poincaré coordinate, $\mathrm{AdS}_{2}$ metric takes the form $d s^{2}=\frac{L_{2}^{2}}{\rho^{2}}\left(d t^{2}+d \rho^{2}\right)$ which implies $g_{u u}=\frac{L_{2}^{2}}{\rho^{2}}\left(t^{\prime 2}+\rho^{\prime 2}\right)=\frac{L_{2}^{2}}{\epsilon^{2}}$. The solution of this equation gives the equation of the boundary curve as $\rho(u) \simeq$ $\epsilon t^{\prime}(u)$ in the small $\epsilon$ limit, where the prime denotes the derivative with respect to the boundary time $u$. Then the extrinsic curvature at the boundary takes the following form:

$$
K=\frac{t^{\prime}\left(t^{\prime 2}+\rho^{\prime 2}+\rho \rho^{\prime \prime}\right)-\rho \rho^{\prime} t^{\prime \prime}}{L_{2}\left(t^{\prime 2}+\rho^{\prime 2}\right)^{\frac{3}{2}}}=\frac{1}{L_{2}}\left(1+\epsilon^{2} \operatorname{sch}(t, u)\right),
$$

where

$$
\operatorname{sch}(t, u)=-\frac{1}{2}\left(\frac{t^{\prime \prime}}{t^{\prime}}\right)^{2}+\left(\frac{t^{\prime \prime}}{t^{\prime}}\right)^{\prime}
$$

Thus, the boundary action can be written as

$$
\mathfrak{S}_{\mathrm{bdy}}^{1}=-\frac{r_{0}^{2}}{G_{4}} \int_{0}^{\beta} d u \phi_{b} \operatorname{sch}(t, u) .
$$

The corresponding partition function, which includes quantum corrections, takes into account the path integrals over all possible boundaries $t(u)$ up to $S L(2, R)$ identification and is given by [10,44]

$$
Z_{\text {sch }}\left(\phi_{b}, \beta\right)=\left(\frac{\hat{\phi}_{b}}{\beta}\right)^{\frac{3}{2}} e^{\frac{2 \pi^{2} \hat{\phi}_{b}}{\beta}},
$$

where $\hat{\phi}_{b}=\frac{r_{0}^{2}}{G_{4}} \phi_{b}$. The entropy derived from this partition function provides the entropy of a near-extremal black hole above extremality and is [44]

$$
\delta S=\frac{3}{2}+4 \pi^{2} \hat{\phi}_{b} T+\frac{3}{2} \log \left(T \hat{\phi}_{b}\right),
$$

where we take the identification of the temperature $T$ with the inverse of the periodicity of the boundary time as $T=1 / \beta$. Now we need to interpret the value of $\phi_{b}$ in terms of the parameter of the near-extremal black hole.

The generic solution for the equation for the dilaton derived from Eq. (3.21) is given by $\Phi=r$, which at the horizon of the extremal solution takes the value $\Phi=r_{0}$. Thus, at any point near the horizon it takes the value given by $\Phi=r_{0}(1+\phi)$ as Eq. (3.36). In global coordinates, we fix the position of the boundary separating $\mathrm{NH}$ and $\mathrm{FH}$ as $r=r_{0}+r_{b}$. Dilaton takes the constant value $\Phi=r=$ $r_{0}\left(1+\frac{\phi_{b}}{\epsilon}\right)$ at the boundary. Thus, at the boundary $\phi_{b}=\frac{\left(r-r_{0}\right) \epsilon}{r_{0}}$. Using the relation between the global coordinate and the Poincaré patch (3.29), one can see that $\phi_{b}=\frac{L_{2}^{2}}{r_{0}}=r_{0}$ at the boundary where $\rho \rightarrow \epsilon$ [44]. Thus, we find from Eq. (3.43) that

$$
\delta S=\frac{3}{2}+\frac{4 \pi^{2}}{G_{4}} r_{0}^{3} T+\frac{3}{2} \log \left(\frac{r_{0}^{3} T}{G_{4}}\right) .
$$

The near-extremal entropy is

$S_{\mathrm{NE}}=S_{0}+\delta S=\frac{\pi r_{0}^{2}}{G_{4}}\left(1+4 \pi T r_{0}\right)+\frac{3}{2} \log \left(\frac{r_{0}^{3} T}{G_{4}}\right)+\frac{3}{2}$,

which is now manifestly independent of the boundary.

\section{NEAR-EXTREMAL LIMIT AND F-DUALITY}

At this point it is worth reviewing what we have calculated thus far. We started with the bosonic sector of the $\mathcal{N}=2$ supergravity and dimensionally reduced that to two dimensions for a very specific case. Our main goal is to probe the fate of F-duality in this kind of scenario. Therefore, it is imperative that we keep track of the charge dependence in all our variables. As can be seen from the two-dimensional effective action (3.22), information of the initial charge configuration is embedded in the coefficient denoted by $\Theta(\mathcal{Q})$, which is fixed for a specific supergravity theory under consideration. 
As summarized briefly in Sec. II, Freudenthal duality in the physics literature is defined using [27-30] an antiinvolutive operator on the moduli space of the black hole charges as

$$
\hat{\mathcal{Q}}^{\mathcal{A}}=\Omega_{\mathcal{A B}} \frac{\partial \sqrt{|\Delta(\mathcal{Q})|}}{\partial \mathcal{Q}^{\mathcal{B}}} .
$$

With $\Delta(\mathcal{Q})$ being an invariant quartic polynomial and $\Omega$ is a $2 m \times 2 m$ symplectic metric $\left(\Omega^{T}=-\Omega, \Omega^{2}=-1\right)$, with $m$ being the number of vector fields of the theory. Where the Bekenstein-Hawking (BH) entropy can be written as

$$
S=\pi \sqrt{|\Delta(\mathcal{Q})|}
$$

This duality is ill defined for small black holes where $|\Delta(\mathcal{Q})|=0$. Contrary to the usual U-duality, where the black hole charges transform linearly, F-duality is highly nonlinear, as evident from the quartic polynomial defined above, although both of them conserve the BH entropy. In fact, following [31-33] one can show that the critical points of the black hole potential are also preserved under F-duality. Freudenthal duality and its generalities also appear in various other contexts in the literature, such as in the study of multicentered black holes [34] and in the formulation of gauge theories with symplectic scalar manifolds [30]. For other interesting avenues one may refer to $[35,36]$.

We now return to the present content. As discussed at the end of Sec. II, F-duality entails that

$$
\begin{aligned}
\pi \hat{\mathcal{Q}}^{M} & =\Omega^{M N} \frac{\partial S(\mathcal{Q})}{\partial \mathcal{Q}^{N}}, \\
\hat{\hat{\mathcal{Q}}} & =-\mathcal{Q}, \quad \text { and } \quad S(\hat{\mathcal{Q}})=S(\mathcal{Q}),
\end{aligned}
$$

where $\Omega^{M N}$ is a $2(n+1) \times 2(n+1)$ symplectic matrix with $\Omega^{T}=-\Omega$ and $\Omega^{2}=-I$. We have already derived that for our case as

$$
\begin{aligned}
S_{0}(\mathcal{Q}) & =\frac{2 \pi \Theta(\mathcal{Q})}{G_{4}} \\
& =\frac{\pi}{3 p^{0}} \sqrt{\frac{4}{3} \frac{\left(3 D-p^{0} q_{a} p^{a}\right)^{3}}{D}-9\left(p^{0}(p \cdot q)-2 D\right)^{2}} .
\end{aligned}
$$

One can now check to ensure that $S_{0}(\mathcal{Q})$ remains invariant as advertised under Eq. (4.3) with $S(\mathcal{Q})=S_{0}(\mathcal{Q})$. The anti-involution of this operation can be checked starting with Eq. (4.4) as the generating function for the F-dualization. In fact, continual use of extremal entropy to generate F-duality indeed preserves the near-extremal entropy $S_{\mathrm{NE}}$ as well.
The current setup leads us to ask a question about the fate of F-duality invariance beyond the extremal limit. Naively, one might consider that F-duality can also be generated by the complete near-extremal entropy [assuming that $S(\mathcal{Q})=S_{\mathrm{NE}}$ ]. As pointed out in Eq. (3.45), the boundary condition at the overlapping region of the $\mathrm{NH}$ and the $\mathrm{FH}$ forces the complete entropy into a nonlinear dependence on $\Theta(\mathcal{Q})$. One can now check by using Eq. (3.45) in Eq. (4.3) and taking

$$
\begin{aligned}
S(\mathcal{Q})= & S_{\mathrm{NE}}=\frac{2 \pi \Theta(\mathcal{Q})}{G_{4}}(1+4 \pi T \sqrt{2 \Theta(\mathcal{Q})}) \\
& +\frac{3}{2} \log \left(2 \Theta(\mathcal{Q}) \sqrt{2 \Theta(\mathcal{Q})} \frac{T}{G_{4}}\right)+\frac{3}{2},
\end{aligned}
$$

with

$\Theta(\mathcal{Q})=\frac{G_{4}}{6 p^{0}} \sqrt{\frac{4}{3} \frac{\left(3 D-p^{0} q_{a} p^{a}\right)^{3}}{D}-9\left(p^{0}(p \cdot q)-2 D\right)^{2}}$.

We find that, in this case, F-duality mapping is no longer a symmetry of entropy. This mapping also fails to retain the anti-involution property. Since the quantum correction appearing at the nonzero temperature diverges at the zero temperature limit, one might consider ignoring the quantum correction discussed in Eq. (3.45) and generate the F-duality through the classical piece of the nearextremal entropy. It can be checked to see that, even in that case, the F-duality fails to be a symmetry of the entropy. Therefore, we conclude that F-duality continues to hold when the transformation is generated by the extremal entropy, although it does not hold when it is generated by the near-extremal entropy with or without the quantum correction.

\section{CONCLUSION}

In this paper, we have studied the invariance of the entropy of a near-extremal, four-dimensional black hole in $\mathcal{N}=2$ ungauged supergravity under Freudenthal duality (F-duality), which is an anti-involutional mapping of a black hole charge vector. We have considered spherically symmetric, dyonically charged black holes in asymptotically flat space. When the black hole is extremal and supersymmetric, the entropy is F-duality invariant. As we approach the near-extremal limit, the theory is well captured by JT gravity upon a dimensional reduction on $S^{2}$. Using JT gravity theory as our apparatus, we find that the invariance of entropy of a near-extremal black hole depends on how we define the duality itself. Originally, for a supersymmetric, extremal black hole, the duality derives a new set of charge vectors by taking derivatives of the extremal, supersymmetric entropy $S_{0}(\mathcal{Q})$ with respect to the corresponding charges. Considering this definition 
of F-duality, we find a new set of charges. We see that the entropy corresponding to the new charges is the same as the near-extremal entropy of the original charges, i.e., the entropy is F invariant. However, when we tweak the mapping of the charges, to be explicit, if we define the new set of charges as the variation of the nearextremal entropy $S_{\mathrm{NE}}$, we find that in this case near-extremal entropy is not $\mathrm{F}$ invariant. Therefore, we see that the effect of F-duality on the entropy of a near-extremal black hole depends on how we define the mapping of the charge vectors.

F-duality in supergravity is broadly studied for extremal, supersymmetric black holes. In this paper, we have extended this study to a near-extremal black hole as a deviation of an extremal, supersymmetric black hole. It would be interesting to analyze the effect of Freudenthal duality on extremal, nonsupersymmetric, and nonextremal black holes in both ungauged and gauged supergravity, with and without truncation of the fermions. It would also be interesting to analyze the invariance of entropy under F-duality for a rotating black hole solution in supergravity. An extremal black hole in gauged supergravity is Freudental invariant [54]. Thus, it would be interesting to analyze the effect of Freudenthal duality on a nearextremal black hole in gauged supergravity. In this work, we have considered a double-extremal solution to study the near-extremal limit of F-duality, where we have benefited from the constant moduli fields but have not had other parameters to control the duality transformation. The inclusion of nonconstant moduli could give us a better handle on how F-duality acts away from extremality. Therefore, it would be interesting to generalize our study to a near-extremal black hole without considering the double-extremal limit. We reserve these considerations for future endeavors.

\section{ACKNOWLEDGMENTS}

We would like to thank Nabamita Banerjee, Prasanta K. Tripathy, Robert de Mello Koch, and Vishnu Jejjala for the helpful comments on our initial draft. We also would like to thank Muktajyoti Saha for the many useful discussions at many stages of the project. The work of A. C. and T. M. is supported by Simons Foundation Grant No. 509116 and by the South African Research Chairs Initiative of the Department of Science and Technology and the National Research Foundation.

\section{APPENDIX: BACKGROUND SOLUTION}

In this appendix we discuss the attractor solutions while following [55]. This rather simple case consists of three complex scalar and four gauge fields:

$$
\begin{aligned}
x^{1} & =S, \quad x^{2}=T, \quad x^{3}=U, \quad \text { and } \\
D_{a b c} & = \begin{cases}1 / 6 & \forall a \neq b \neq c, \\
0 & \text { otherwise. }\end{cases}
\end{aligned}
$$

The justification of this naming comes partially from the fact that in these cases the prepotential can be written as $F=$ STU. The prepotential $F$ defined earlier in Eq. (2.2) boils down simply to

$$
F=\frac{X^{1} X^{2} X^{3}}{X^{0}}
$$

Since we are taking the double-extremal black hole, we have a much simpler tractable setup, as the scalars $x^{a}=\left(x_{1}^{a}+i x_{2}^{a}\right)$ take a constant value over all the spacetime [56]. $a$ denotes the number of vector multiplets in the case where STU $a=3$. For a supersymmetric $D 0-D 2-D 4-D 6$ system, the attractor values are [48]

$$
\begin{gathered}
x_{1}^{a}=\frac{3}{2} \frac{\tilde{x}^{a}}{p^{0}\left(\Delta_{c} \tilde{x}^{c}\right)}\left(p^{0}(p \cdot q)-2 D\right)+\frac{p^{a}}{p^{0}}, \\
x_{2}^{a}=\frac{3}{2} \frac{\tilde{x}^{a}}{\left(\Delta_{c} \tilde{x}^{c}\right)} \frac{S}{\pi}
\end{gathered}
$$

with $q_{0}$ and $q_{a}$ denoting $D 0$ - and $D 2$-brane charges, respectively, while $p^{0}$ and $p^{a}$ denote $D 6$ - and $D 4$-brane charges, respectively. The entropy $S$ is [48]

$$
S=\frac{\pi}{3 p^{0}} \sqrt{\frac{4}{3}\left(\Delta_{a} \tilde{x}^{a}\right)^{2}-9\left(p^{0}(p . q)-2 D\right)^{2}}
$$

and $\tilde{x}^{a}$ is the real solution of $\Delta_{a}=D_{a b c} \tilde{x}^{b} \tilde{x}^{c}=$ $3 D_{a}-p^{0} q_{a}$, with $D_{a}=D_{a b c} p^{b} p^{c}$.

Considering an ansatz in which $\tilde{x}^{a}=\sqrt{\frac{3 D-p^{0} q_{a} p^{a}}{D}} p^{a}$, we see that the entropy can be written as [37]

$S=\frac{\pi}{3 p^{0}} \sqrt{\frac{4}{3} \frac{\left(3 D-p^{0} q_{a} p^{a}\right)^{3}}{D}-9\left(p^{0}(p \cdot q)-2 D\right)^{2}}$. 
[1] C. Teitelboim, Gravitation and Hamiltonian structure in two space-time dimensions, Phys. Lett. B 126, 41 (1983).

[2] R. Jackiw, Lower dimensional gravity, Nucl. Phys. B252, 343 (1985).

[3] J. Maldacena, D. Stanford, and Z. Yang, Conformal symmetry and its breaking in two-dimensional nearly anti-de Sitter space, Prog. Theor. Exp. Phys. (2016) $12 \mathrm{C} 104$.

[4] A. Almheiri and J. Polchinski, Models of $\mathrm{AdS}_{2}$ backreaction and holography, J. High Energy Phys. 11 (2015) 014.

[5] S. Sachdev and J. Ye, Gapless Spin-Fluid Ground State in a Random Quantum Heisenberg Magnet, Phys. Rev. Lett. 70, 3339 (1993).

[6] A. Kitaev and S. J. Suh, The soft mode in the Sachdev-YeKitaev model and its gravity dual, J. High Energy Phys. 05 (2018) 183.

[7] J. Maldacena and D. Stanford, Remarks on the Sachdev-YeKitaev model, Phys. Rev. D 94, 106002 (2016).

[8] K. Jensen, Chaos in $\mathrm{AdS}_{2}$ Holography, Phys. Rev. Lett. 117, 111601 (2016).

[9] G. Sárosi, $\mathrm{AdS}_{2}$ holography and the SYK model, Proc. Sci., Modave2017 (2018) 001 [arXiv:1711.08482].

[10] N. Banerjee, T. Mandal, A. Rudra, and M. Saha, Equivalence of JT gravity and near-extremal black hole dynamics in higher derivative theory, J. High Energy Phys. 01 (2022) 124.

[11] H. Rathi and D. Roychowdhury, Holographic JT gravity with quartic couplings, J. High Energy Phys. 10 (2021) 209.

[12] J. Engelsöy, T. G. Mertens, and H. Verlinde, An investigation of $\mathrm{AdS}_{2}$ backreaction and holography, J. High Energy Phys. 07 (2016) 139.

[13] P. Saad, S. H. Shenker, and D. Stanford, JT gravity as a matrix integral, arXiv:1903.11115.

[14] D. Stanford and E. Witten, JT gravity and the ensembles of random matrix theory, Adv. Theor. Math. Phys. 24, 1475 (2020).

[15] C. V. Johnson, Explorations of nonperturbative JackiwTeitelboim gravity and supergravity, Phys. Rev. D 103, 046013 (2021).

[16] C. V. Johnson, Jackiw-Teitelboim supergravity, minimal strings, and matrix models, Phys. Rev. D 103, 046012 (2021).

[17] F. Larsen, A nAttractor mechanism for $\mathrm{nAdS}_{2} / \mathrm{nCFT}_{1}$ holography, J. High Energy Phys. 04 (2019) 055.

[18] A. Castro, F. Larsen, and I. Papadimitriou, 5D rotating black holes and the $\mathrm{nAdS}_{2} / \mathrm{nCFT}_{1}$ correspondence, J. High Energy Phys. 10 (2018) 042.

[19] A. Castro and E. Verheijden, Near-AdS 2 spectroscopy: Classifying the spectrum of operators and interactions in $\mathcal{N}=2$ 4D supergravity, Universe 7, 475 (2021).

[20] S. Ferrara, R. Kallosh, and A. Strominger, $N=2$ extremal black holes, Phys. Rev. D 52, R5412 (1995).

[21] S. Ferrara, G. W. Gibbons, and R. Kallosh, Black holes and critical points in moduli space, Nucl. Phys. B500, 75 (1997).

[22] P. K. Tripathy and S. P. Trivedi, Non-supersymmetric attractors in string theory, J. High Energy Phys. 03 (2006) 022.

[23] P. Dominic, T. Mandal, and P. K. Tripathy, Multiple singlecentered attractors, J. High Energy Phys. 12 (2014) 158.

[24] K. Goldstein, V. Jejjala, and S. Nampuri, Hot attractors, J. High Energy Phys. 01 (2015) 075.
[25] K. Goldstein, V. Jejjala, and S. Nampuri, The hot attractor mechanism: Decoupling without deep throats, J. High Energy Phys. 04 (2016) 026.

[26] H. Freudenthal, Beziehungen der e7 und e8 zur oktavenebene. I, Indag. Math. (Proceedings) 57, 218 (1954).

[27] L. Borsten, D. Dahanayake, M. J. Duff, and W. Rubens, Black holes admitting a Freudenthal dual, Phys. Rev. D 80, 026003 (2009).

[28] M. Günaydin, Lectures on spectrum generating symmetries and U-duality in supergravity, extremal black holes, quantum attractors and harmonic superspace, in The Attractor Mechanism, Springer Proceedings in Physics Vol. 134, edited by S. Bellucci (Springer, New York, 2010), pp. 31-84.

[29] L. Borsten, M. J. Duff, S. Ferrara, A. Marrani, and W. Rubens, Explicit orbit classification of reducible Jordan algebras and Freudenthal triple systems, Commun. Math. Phys. 325, 17 (2014).

[30] A. Marrani, C.-X. Qiu, S.-Y. D. Shih, A. Tagliaferro, and B. Zumino, Freudenthal gauge theory, J. High Energy Phys. 03 (2013) 132.

[31] S. Ferrara, A. Marrani, and A. Yeranyan, Freudenthal duality and generalized special geometry, Phys. Lett. B 701, 640 (2011).

[32] S. Ferrara, A. Marrani, E. Orazi, and M. Trigiante, Dualities near the horizon, J. High Energy Phys. 11 (2013) 056.

[33] A. Marrani, Freudenthal duality in gravity: From groups of type $E_{7}$ to pre-homogeneous spaces, P-Adic Num. Ultrametr. Anal. Appl. 7, 322 (2015).

[34] J. J. Fernández-Melgarejo and E. Torrente-Lujan, $N=2$ SUGRA BPS multi-center solutions, quadratic prepotentials and Freudenthal transformations, J. High Energy Phys. 05 (2014) 081.

[35] L. Borsten, M. J. Duff, S. Ferrara, and A. Marrani, Freudenthal dual Lagrangians, Classical Quantum Gravity 30, 235003 (2013).

[36] D. Klemm, A. Marrani, N. Petri, and M. Rabbiosi, Nonlinear symmetries of black hole entropy in gauged supergravity, J. High Energy Phys. 04 (2017) 013.

[37] A. Marrani, P. K. Tripathy, and T. Mandal, Supersymmetric black holes and Freudenthal duality, Int. J. Mod. Phys. A 32, 1750114 (2017).

[38] P. Meessen, T. Ortin, J. Perz, and C. S. Shahbazi, H-FGK formalism for black-hole solutions of $\mathcal{N}=2, d=4$ and $d=5$ supergravity, Phys. Lett. B 709, 260 (2012).

[39] P. Galli, P. Meessen, and T. Ortin, The Freudenthal gauge symmetry of the black holes of $\mathcal{N}=2, d=4$ supergravity, J. High Energy Phys. 05 (2013) 011.

[40] D. D. K. Chow and G. Compère, Seed for general rotating non-extremal black holes of $\mathcal{N}=8$ supergravity, Classical Quantum Gravity 31, 022001 (2014).

[41] D. D. K. Chow and G. Compère, Black holes in $\mathcal{N}=8$ supergravity from $\mathrm{SO}(4,4)$ hidden symmetries, Phys. Rev. D 90, 025029 (2014).

[42] G. Sárosi, Entropy of nonextremal STU black holes: The F-invariant unveiled, Phys. Rev. D 93, 024036 (2016).

[43] After integrating out the gauge fields [44].

[44] L. V. Iliesiu and G. J. Turiaci, The statistical mechanics of near-extremal black holes, J. High Energy Phys. 05 (2021) 145. 
[45] Once we dimensionally reduce higher-dimensional theory, a dilaton appears in lower-dimensional theory.

[46] P. Nayak, A. Shukla, R. M. Soni, S. P. Trivedi, and V. Vishal, On the dynamics of near-extremal black holes, J. High Energy Phys. 09 (2018) 048.

[47] M. Heydeman, L. V. Iliesiu, G. J. Turiaci, and W. Zhao, The statistical mechanics of near-BPS black holes, J. Phys. A 55, 014004 (2022).

[48] M. Shmakova, Calabi-Yau black holes, Phys. Rev. D 56, R540 (1997).

[49] T. Mandal and P. K. Tripathy, On the uniqueness of supersymmetric attractors, Phys. Lett. B 749, 221 (2015).

[50] This is not the most generic ansatz for dimensional reduction. Considering the most generic ansatz, in lower dimensions, one gets Maxwell-Dilaton gravity theory with an $S O(3)$ Yang-Mills field Iliesiu:2020qvm. Integrating out the YangMills field, one finds a rotational solution with angular momentum $j$ [44]. As our goal is to comment on Freudenthal duality of a spherically symmetric, nonrotating, nearextremal black hole, we can set $j=0$. This is equivalent to considering the ansatz (3.1) for dimensional reduction.
[51] We are considering $G_{\mu \nu}$ to be asymptotically flat.

[52] As we are interested in a fixed charge black hole solution, we need to add a boundary term for the gauge field such as Eq. (3.9) [A. Chamblin, R. Emparan, C. V. Johnson, and R. C. Myers, Charged AdS black holes and catastrophic holography, Phys. Rev. D 60, 064018 (1999)].

[53] A. Sen, Black hole entropy function, attractors and precision counting of microstates, Gen. Relativ. Gravit. 40, 2249 (2008).

[54] D. Klemm, A. Marrani, N. Petri, and M. Rabbiosi, Nonlinear symmetries of black hole entropy in gauged supergravity, J. High Energy Phys. 04 (2017) 013.

[55] K. Behrndt, R. Kallosh, J. Rahmfeld, M. Shmakova, and W. K. Wong, STU black holes and string triality, Phys. Rev. D 54, 6293 (1996).

[56] In a generic case for supersymmetric attractors, one can write the moduli at any point of spacetime in terms of the harmonic functions [T. Mandal and P. K. Tripathy, New supersymmetric black holes in four-dimensional $N=2$ supergravity, Mod. Phys. Lett. A 31, 1650100 (2016)]. 